\title{
Galaxy merging and number vs. apparent magnitude relation for the universe with a time-decaying cosmological term
}

\author{
Z. Komiya ${ }^{1}$, K. Kawabata ${ }^{1}$, K. Hirano ${ }^{1}$, H. Bunya ${ }^{1}$, and N. Yamamoto ${ }^{2}$ \\ ${ }^{1}$ Department of Physics, Tokyo University of Science, Shinjuku-ku, Tokyo, 162-8601, Japan \\ e-mail: zen_komiya@ybb.ne.jp; kawabata@rs.kagu.tus.ac.jp \\ 2 Grid Technology Research Center, National Institute of Advanced Industrial Science and Technology \\ Ibaraki 305-8568, Japan
}

Received 25 October 2005 / Accepted 13 December 2005

\section{ABSTRACT}

\begin{abstract}
Aims. An attempt is made to constrain the values of the cosmological parameters together with the galaxy merging factor $\eta$ on the basis of a comparison between the observed galaxy number counts vs. their apparent magnitudes relation ( $N-m$ relation) with those theoretically constructed for the universe with a time-decaying cosmological term $\Lambda$.

Methods. We assume that the galaxy number density evolution can be represented sufficiently well by a function of the redshift $z$ of the form $\phi^{*}(z) \propto(1+z)^{\eta}$. Three variations of the cosmological term with time $\tau$ are considered, (1) $\Lambda \propto \tau^{-l}$, (2) $\Lambda \propto a^{-m}$ with $a$ being the scale factor, and (3) $\Lambda \propto H^{n}$ with $H$ the Hubble parameter. The optimum ranges for the decaying parameters $(l, m$, and $n)$, the density parameters $\Omega_{\Lambda, 0}$ and $\Omega_{\mathrm{m}, 0}$, as well as $T_{\mathrm{mg}}$ (the timescale for the merger of a pair of galaxies) and the redshift $z_{\mathrm{mg}}$ for the first onset of galaxy merger are sought based on statistical analysis using likelihood functions given by $\chi^{2}$ evaluations.

Results. In the case of the type I models, for instance, we find that $l=0.75_{-0.75}^{+0.55}, T_{\mathrm{mg}}=0.3 \mathrm{Gyr}, z_{\mathrm{mg}}=3, \Omega_{\Lambda, 0}=0.71_{-0.09}^{+0.17}$ and $\Omega_{\mathrm{m}, 0}=0.29_{-0.17}^{+0.09}$, from which a cosmic age of $17.2_{-1.2}^{+1.3} \mathrm{Gyr}$ results. These model parameters lead to $\eta=1.73_{-0.07}^{+0.14}$. As a consistency check, we have also carried out computations of the cosmic microwave background radiation (CMBR) spectrum, and have made comparisons with WMAP measurements. We found that it is necessary to somewhat modify the parameter values obtained above on account of the high sensitivity of $\eta$ to the value of $T_{\mathrm{mg}}$. The final model that was found to account for both the observed $N-m$ relation and the WMAP measurements of the CMBR spectrum is as follows: $z_{\mathrm{mg}}=3.0, T_{\mathrm{mg}}=0.2 \mathrm{Gyr}, l=0.04, \Omega_{\Lambda, 0}=0.77, \eta=2.2269$. The age of this model universe is $14.6 \mathrm{Gyr}$, which is still sufficiently high to cope with the "new" cosmic age problem.
\end{abstract}

Key words. cosmological parameters - Galaxy: general - cosmic microwave background

\section{Introduction}

The existence of Einstein's cosmological term $\Lambda$ is now accepted by a majority of investigators based on observations of the magnitudes and corresponding redshifts of several highredshift type Ia supernovae (Riess et al. 1998; Perlmutter et al. 1999a). Nevertheless, the origins and the physical nature of Einstein's cosmological term are not yet clear, and there does not seem to be any a priori basis to assume its constancy with time. In fact, the possibility of time variation of the cosmological term has actually been investigated by a number of authors (see for example, Peebles \& Ratra 2003, for a recent review). This line of research may be categorized into three types: i) scalar field models (Ferreira \& Joyce 1997; Coble et al. 1997; Perlmutter et al. 1999b; Viana \& Liddle 1998; Jackson \& Dodgson 1998; Frieman \& Waga 1998; Díaz-Rivera \& Pimentel 1999; Podariu \& Ratra 2000; Aurich $\&$ Steiner 2003; Barreiro et al. 2003); ii) explicit time decaying cosmological models (Overduin \& Cooperstock 1998); and iii) modified equation-of-state models (Chiba et al. 1997;
Turner \& White 1997; Caldwell et al. 1998; Huterer \& Turner 2001; Dev et al. 2003). Among the studies belonging to category i) are the oscillating scalar field models, in which the scalar field exhibits an oscillatory dissipation with time towards the present epoch (Morikawa 1990; Kashino \& Kawabata 1994; Kawabata et al. 2002).

In this work, we investigate some typical models of category ii). The reason for considering these models is that they are a straightforward extension of the Friedmann-Lemaitre model incorporating Einstein's cosmological term, which is the current standard model (see Overduin \& Cooperstock 1998, for a general review). It is to be noted that category ii)-models have been investigated from various points-of-view of observational cosmology, such as large scale structure formation (Silveira \& Waga 1994), type Ia supernovae observations (Alcaniz \& Maia 2003), angular size vs. redshift relationship (Cunha et al. 2002), cosmic microwave background radiation (CMBR) spatial power spectra (Silveira \& Waga 1997), gravitational lens effects (Bloomfield Torres \& Waga 1996; Waga \& Miceli 1999), etc. 
Another typical classical test for observational cosmology is the number count vs. apparent magnitude relation or the $N-m$ relation for galaxies, which has been widely employed and pursued observationally as well as theoretically by Yoshii \& Takahara (1988); Fukugita et al. (1990); Yoshii \& Peterson (1991); Lacey \& Silk (1993); Shimasaku \& Fukugita (1998), ever since Sandage $(1961,1965)$ introduced the method. However, in the case of the $B_{\mathrm{J}}$ band data of the $N-m$ relation, we have had to deal with the so-called "faint blue galaxy problem" (hereafter referred to as FBGP), wherein the galaxy number counts obtained from observations for magnitudes greater than $\sim 24$ tend to be higher roughly by a factor of 10 than those predicted theoretically (Tyson 1988; Griffiths et al. 1994; Driver \& Phillipps 1996; Gardner et al. 1996; Mihara 2001). Various astrophysical hypotheses have therefore been proposed to resolve this problem, such as the effects of galaxy mergers (Koo 1986), chemical evolution of galaxies (Yoshii \& Takahara 1988; Guiderdoni \& Rocca-Volmerange 1990), internal absorption (Wang 1991), the existence of dwarf galaxies (Abraham et al. 1996), the choice of the redshift value for galaxy formation (Gardner 1998), etc. From the view-point of cosmology, however, the effects of galaxy merger appears to be the primary cause for the FBGP. Today, hierarchical clusterings that take place in CDM models are generally accepted to be the crucial mechanism for large scale structure formation (Peacock et al. 2001). In fact, a large number of observations have demonstrated the presence of active galaxy mergers (Patton et al. 2002). One very practical way to incorporate the effects of such galaxy mergers into the $N-m$ relation would be to express the galaxy number density evolution as a function of redshift $z$ (Rocca-Volmerange \& Guiderdoni 1990; Broadhurst et al. 1992; Glazebrook et al. 1994). However, another important parameter is $T_{\mathrm{mg}}$, the timescale for a galaxy merger, which is usually believed to be on the order of $0.5 \mathrm{Gyr}$ (Chatterjee 1993; Patton et al. 1997; Barnes 1988; Cohn et al. 2001; Gottlöber et al. 2002; Lu \& Wei 2003; Hernandez \& Lee 2004; Conselice et al. 2003), although the values that have been proposed by different authors differ from each other depending on their definitions for initiation and the completion of the galaxy merging process.

Another crucial test would be to check the lower limit for the cosmic age, which we adopt to be $\sim 13 \mathrm{Gyr}$ based on stellar age determinations (see Sect. 4.1 for a detailed discussion). For instance, the flat Friedmann-Lemaitre model coupled with the use of WMAP measurements of the CMBR anisotropy (Spergel et al. 2003; Hinshaw et al. 2003; Kogut et al. 2003) and observations of type Ia supernovae (Riess et al. 1998; Perlmutter et al. 1999a) have yielded $\Omega_{\mathrm{m}, 0} \simeq 0.3$ for the matter density parameter and $\Omega_{\Lambda, 0} \simeq 0.7$ for the dark energy density parameter of the present epoch. Using $H_{0}=70 \mathrm{~km} \mathrm{~s}^{-1} \mathrm{Mpc}^{-1}$, the age of our universe is found to be $13.5 \mathrm{Gyr}$, which is sufficiently high to circumvent the well-known "old cosmic age problem", or the relatively large discrepancy between theoretical model ages and the lower bound for the cosmic age ( $\sim 13 \mathrm{Gyr})$ inferred from observations of globular clusters (Chaboyer et al. 1996; Krauss \& Chaboyer 2003). However, we cannot yet rule out the possibility that some metal-poor stars may well be older than 13 Gyr (Cowan et al. 1997, 1999;
Johnson \& Bolte 2001; Westin et al. 2000; Rengel et al. 2002; Schatz et al. 2002; Sneden et al. 2003), although uncertainties associated with the observations are rather large. Obviously, the age indicated by the standard Friedmann-Lemaitre model leaves little margin to allow for this possibility.

The primary purpose of our work is to provide as strong a constraint as possible to the models of category ii), that explicitly incorporate the effects of the time-decaying cosmological term, primarily by making comparisons between observed and theoretical $N-m$ relationships of galaxies as well as by giving consideration to the age of our universe. Specifically, we shall try to eliminate the cosmic age problem and alleviate the FBGP by carrying out a systematic study of the $N-m$ relations produced for category ii) models. It must be noted that a prototype study along this line of work has been carried out using category i)-type models with a scalar field by Yoshii \& Sato (1992). Furthermore, taking into account the effects of galaxy merger in terms of the merging parameter $\eta$, which was originally introduced into the galaxy number evolution model by Rocca-Volmerange \& Guiderdoni (1990), we shall estimate not only the value of $\eta$ but also the merging timescale $T_{\mathrm{mg}}$.

The validity of the derived best-fit model will further be assessed by comparing the theoretical spectra of the CMBR computed for a grid of type I models including the one derived from the $N-m$ relation analysis with the WMAP measurements. We shall then provide a modification to the best-fit model if necessary, in order to obtain a better model and to obtain one that can account not only for the observed $N-m$ relation but also WMAP measurements of the CMBR spectrum.

The structure of this paper is as follows. Section 2 briefly describes the basic equations for the models with timedecaying cosmological terms with reference to the previous studies. A redefinition of the traditional cosmological parameters, which is inevitable due to the introduction of the decaying cosmological term, is also described. Section 3 delineates the equation for computing the relationship between the galaxy number counts and apparent magnitudes(the $N-m$ relation). In particular, a galaxy merger factor $\eta$ is introduced to describe the galaxy number evolution, and its dependency on the other cosmological parameters are discussed. In Sect. 4.1, we first try to provide some constraints to the model parameters based on a consideration of the cosmic age. Next, we deduce on a best-fit model for each of the three types by comparing computed $N-m$ relations with observational data. Finally, we refine the values of the parameters specifying the best-fit type I model by further testing against the WMAP measurements of the CMBR anisotropy spectrum. The last section summarizes our results.

\section{2. $\wedge$ decaying models}

\subsection{Basic equations}

To derive the evolution equation for the universe with the time-decaying cosmological term $\Lambda$, we begin with Einstein's gravitational equations under homogeneous and isotropic 
conditions:

$G_{\mu \nu}+\Lambda g_{\mu \nu}=\frac{8 \pi G}{c^{4}} T_{\mu \nu}$

$G_{\mu \nu} \equiv R_{\mu \nu}-\frac{1}{2} R g_{\mu \nu}$

where $G_{\mu \nu}$ is the well-known Einstein tensor, $\Lambda$ the cosmological term, $R$ the scalar curvature, $R_{\mu v}$ the Ricci tensor and $T_{\mu \nu}$ the energy-momentum tensor, respectively. $c$ and $G$ denote the speed of light and Newton's gravitational constant, respectively. We also define an effective energy momentum tensor $\tilde{T}_{\mu v}$ such that:

$\tilde{T}_{\mu \nu} \equiv T_{\mu \nu}-\frac{c^{4} \Lambda(t)}{8 \pi G} g_{\mu \nu}$

This implies that the dark energy is included in the source term of Einstein's equation. If $\tilde{T}_{\mu \nu}$ due to the matter and the cosmological constant are assumed to be conserved, we have:

$\Delta^{v} \tilde{T}_{\mu v}=0$.

Under the assumption of the Robertson-Walker line element, the conservation law (4) above yields the following equations (Overduin \& Cooperstock 1998):

$\frac{\mathrm{d}}{\mathrm{d} a}\left(a^{3 \gamma} \rho\right)=-\frac{c^{2} a^{3 \gamma}}{8 \pi G} \frac{\mathrm{d} \Lambda}{\mathrm{d} a}$

$\frac{\mathrm{d}^{2} a}{\mathrm{~d} \tau^{2}}=-\frac{1}{2 a}\left(\frac{\mathrm{d} a}{\mathrm{~d} \tau}\right)^{2}-\frac{1}{2 a} \Omega_{\mathrm{K}, 0}-\frac{1}{2 a^{3}} \Omega_{\mathrm{r}, 0}+\frac{3 a}{2} \Omega_{\Lambda, 0} \frac{\Lambda}{\Lambda_{0}}$

with the time variable expressed in units of Hubble time:

$\tau \equiv H_{0} t$

where we have defined the following parameters:

$\rho_{\text {crit }} \equiv \frac{3 H^{2}}{8 \pi G}$

$\Omega_{\Lambda} \equiv \frac{c^{2} \Lambda}{3 H^{2}}$

$\Omega_{\mathrm{m}} \equiv \frac{8 \pi G \rho_{\mathrm{m}}}{3 H^{2}}$

$\Omega_{\mathrm{r}} \equiv \frac{8 \pi G \rho_{\mathrm{r}}}{3 c^{2} H^{2}}$

$\Omega_{\mathrm{K}} \equiv \frac{c^{2} K}{a^{2} H^{2}}$

with $\rho_{\mathrm{m}}$ being the mass density of the matter, $\rho_{\mathrm{r}}$ the energy density of radiation and $K$ the sign of curvature $( \pm 1$ or 0$)$. The exponent $\gamma$ used above assumes values between 0 and 1 , although in this work, we adopt $\gamma=1$ since we assume the presence of dust-like matter. Other symbols have their usual meanings. It must be mentioned that Eq. (5) signifies the condition of conservation of energy including that of the cosmological term as has been previously mentioned, which in turn implies that the creation of matter takes place at the expense of the cosmological term. Note also that the cosmological constant $\Lambda$ employed here is a function of time $t$.
In the present work, we consider the following types of variations for $\Lambda$ based on reference (Overduin \& Cooperstock 1998):

type I: $\Lambda=\frac{3 H_{0}^{2} \Omega_{\Lambda, 0} \tau_{0}^{l}}{c^{2}} \tau^{-l}$

type II: $\Lambda=\frac{3 H_{0}^{2} \Omega_{\Lambda, 0}}{c^{2}} a^{-m}$

type III: $\Lambda=\frac{3 \Omega_{\Lambda, 0} H_{0}^{2-n}}{c^{2}} H^{n}$

where a parameter with subscript 0 indicates its present-day value.

\subsection{Redefinition of some cosmological parameters}

In order to make the cosmological term decay with time, we must redefine the cosmological parameters $\Omega_{\Lambda}, \Omega_{\mathrm{m}}$ and $\Omega_{\mathrm{K}}$ as shown below:

type I

$\tilde{\Omega}_{\Lambda}=\Omega_{\Lambda, 0} \frac{H_{0}^{2}}{H(\tau)^{2}}\left(\frac{\tau_{0}}{\tau}\right)^{l}$

$\tilde{\Omega}_{\mathrm{m}}=\Omega_{\mathrm{m}, 0} \frac{H_{0}^{2}}{H(\tau)^{2}}$

$$
\times\left(1-\frac{\Omega_{\Lambda, 0}}{\Omega_{\mathrm{m}, 0}} l \tau_{0}^{l} \int_{\tau}^{\tau_{0}} a^{3 \gamma}(\tau) \tau^{-l-1} \mathrm{~d} \tau\right)
$$

$\tilde{\Omega}_{\mathrm{K}}=\Omega_{K, 0} \frac{H_{0}^{2}}{a^{2} H(\tau)^{2}}$

type II

$\tilde{\Omega}_{\Lambda}=\Omega_{\Lambda, 0} \frac{H_{0}^{2}}{H(a)^{2}} a^{-m}$

$\tilde{\Omega}_{\mathrm{m}}=\Omega_{\mathrm{m}, 0} \frac{H_{0}^{2}}{H(a)^{2}} a^{-3 \gamma}$

$$
\times \begin{cases}\left\{1+\left(a^{3 \gamma-m}-1\right) \frac{\Omega_{\Lambda, 0}}{\Omega_{\mathrm{m}, 0}} \frac{m}{3 \gamma-m}\right\} & (3 \gamma \neq m) \\ \left(1+m \frac{\Omega_{\Lambda, 0}}{\Omega_{\mathrm{m}, 0}} \ln a\right) & (3 \gamma=m)\end{cases}
$$

$\tilde{\Omega}_{K}=\Omega_{K, 0} \frac{H_{0}^{2}}{a^{2} H(a)^{2}}$

type III

$\tilde{\Omega}_{\Lambda}=\Omega_{\Lambda, 0} \frac{H_{0}^{2}}{H^{2}(a)}\left(\frac{H(a)}{H_{0}}\right)^{n}$

$\tilde{\Omega}_{\mathrm{m}}=\Omega_{\mathrm{m}, 0} \frac{H_{0}^{2}}{H^{2}} a^{-3 \gamma}$

$$
\times\left(1+\frac{\Omega_{\Lambda, 0}}{\Omega_{\mathrm{m}, 0}} n \int_{a}^{a_{0}} a^{3 \gamma}\left(\frac{H(a)}{H_{0}}\right)^{n-1} \frac{\mathrm{d} H(a)}{\mathrm{d} a} \mathrm{~d} a\right)
$$

$\tilde{\Omega}_{\mathrm{K}}=\Omega_{\mathrm{K}, 0} \frac{H_{0}^{2}}{a^{2} H^{2}(a)}$.

The behaviors of the redefined parameters are shown in Fig. 1 for each type. We can clearly see that they gradually deviate from those based on the Friedmann-Lemaitre models as the values of the decay parameters $l, m$, and $n$ become large. Figure 1 

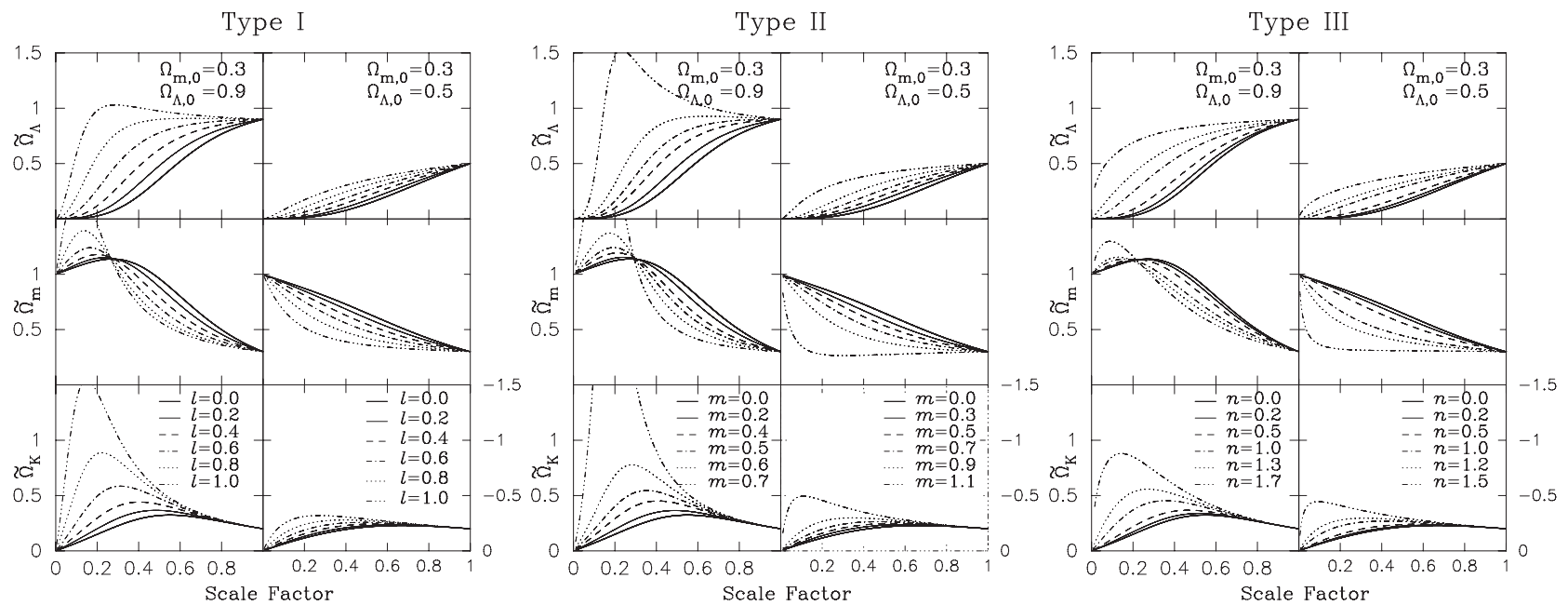

Fig. 1. Time variations of the cosmological parameters for type I, II and III models. The leftmost panel is for the type I model, the middle panel is for the type II models, and the rightmost panel is for the type III models. In each panel, three kinds of density parameters, from top to bottom, $\Omega_{\Lambda}, \Omega_{\mathrm{m}}$, and $\Omega_{\mathrm{K}}$, are shown as functions of the scale factor for the closed models with positive spatial curvatures (the left-hand side diagrams) and for the open models with negative spatial curvatures (the right-hand side diagrams). The present-day values assumed for the computations are as follows. For the closed case, $\Omega_{\Lambda, 0}=0.9, \Omega_{\mathrm{m}, 0}=0.3, \Omega_{\mathrm{K}, 0}=0.2$, while for the open case, $\Omega_{\Lambda, 0}=0.5, \Omega_{\mathrm{m}, 0}=0.3, \Omega_{\mathrm{K}, 0}=-0.2$. Note that the scales for the $\Omega_{\mathrm{K}}$ values of the open cases are indicated on the right-hand side of each relevant diagram for the obvious reason that negative values must be displayed.

indicates that the sign of the curvature parameter is conserved throughout the expansion because of the presence of the matter creation term as shown by Eq. (5). We shall hereafter restrict ourselves only to the flat universe cases, viz., $\Omega_{\mathrm{K}, 0}=0$. Hence, we only have two free cosmological parameters for each type of model, viz., $l, m$, or $n$ on one hand, and $\Omega_{\Lambda, 0}$ on the other.

\subsection{Sets of parameter values to cause the big bang}

As a notable characteristic feature of the $\Lambda$-decaying models, there exist sets of model parameter values that do not give rise to any Big Bang event. In our work, we assume, for the sake of computational convenience, that the Big Bang has occurred as long as the scale factor $a$ of the model universe is less than 0.01 . The domains of the Big Bang models are shown in Fig. 6, the parameter spaces specified by one of the decay parameters $l$, $m$, or $n$ as a function of $\Omega_{\Lambda, 0}$. We notice in Figs. $1-4$ and 10 of Overduin \& Cooperstock (1998) that in the case of type I or type II models, the scale factor tends to bounce back at a certain look-back time without quite reaching 0 (or the Big Bang) as the value of the decay parameter $l$ or $m$ becomes large. Also shown in Fig. 6 are the isochrones corresponding to cosmic ages of $10,12,14,16,18$, and $20 \mathrm{Gyr}$. Note that the different isochrones seemingly fall on the Big Bang boundary since the Big Bang corresponds to a singular point. It must be mentioned here that all type III cases are always associated with the Big Bang.

\section{Apparent magnitude vs. galaxy number count relation}

In order to assess the viability of our models, we chose the galaxy number count vs. the apparent magnitude relation (the $N-m$ relation) as mentioned in the previous section. Here, we closely follow the computational procedure described in Yoshii \& Takahara (1988).

\subsection{Theoretical models}

To compute the theoretical $N-m$ relations, we employ the galaxy count model (Yoshii \& Takahara 1988) which takes into account the luminosity evolution given by Arimoto \& Yoshii (1986) which incorporates the effects of the chemical evolution of galaxies. As in the case of reference (Yoshii \& Takahara 1988), the spectral energy distribution functions of the five morphological Hubble types (E/S0 : SaB : SBc : Scd : Sdm = 0.321:0.281:0.291: $0.045: 0.062$ in number ratio) are explicitly included. However, for the sake of simplicity, in this work, their time variations are assumed to be identical for all the morphological galaxy types. Needless to say, there exist several important astrophysical effects in addition to those of the chemical evolution of galaxies that can affect the $N-m$ relationship, such as, for example, the Eddington bias (Teerikorpi 2004), internal dust absorption (Wang 1991), the way to model excess dwarf galaxies (Abraham et al. 1996), and the choice of the value of the galaxy formation redshift $z_{\mathrm{f}}$ (Gardner 1998). To carry out an analysis from the stand point of cosmology, however, we investigate the effects of galaxy merger, which we have assumed to be the most influential factor based on the results obtained by Gardner (1998) and Totani \& Yoshii (2000).

Signifying the redshift for the epoch when galaxy formation began by $z_{\mathrm{f}}(=5)$, the number count $N(m)$ of galaxies per unit square degree of the sky, whose apparent magnitudes are in the interval $[m-\Delta m / 2, m+\Delta m / 2]$ can be written as

$N(m)=\frac{1}{4 \pi}\left(\frac{\pi}{180}\right)^{2} \sum_{i} \int_{m-\Delta m / 2}^{m+\Delta m / 2} \int_{0}^{z_{\mathrm{F}}} \frac{\mathrm{d} V}{\mathrm{~d} z} \phi_{i}(M) \mathrm{d} z \mathrm{~d} m$. 

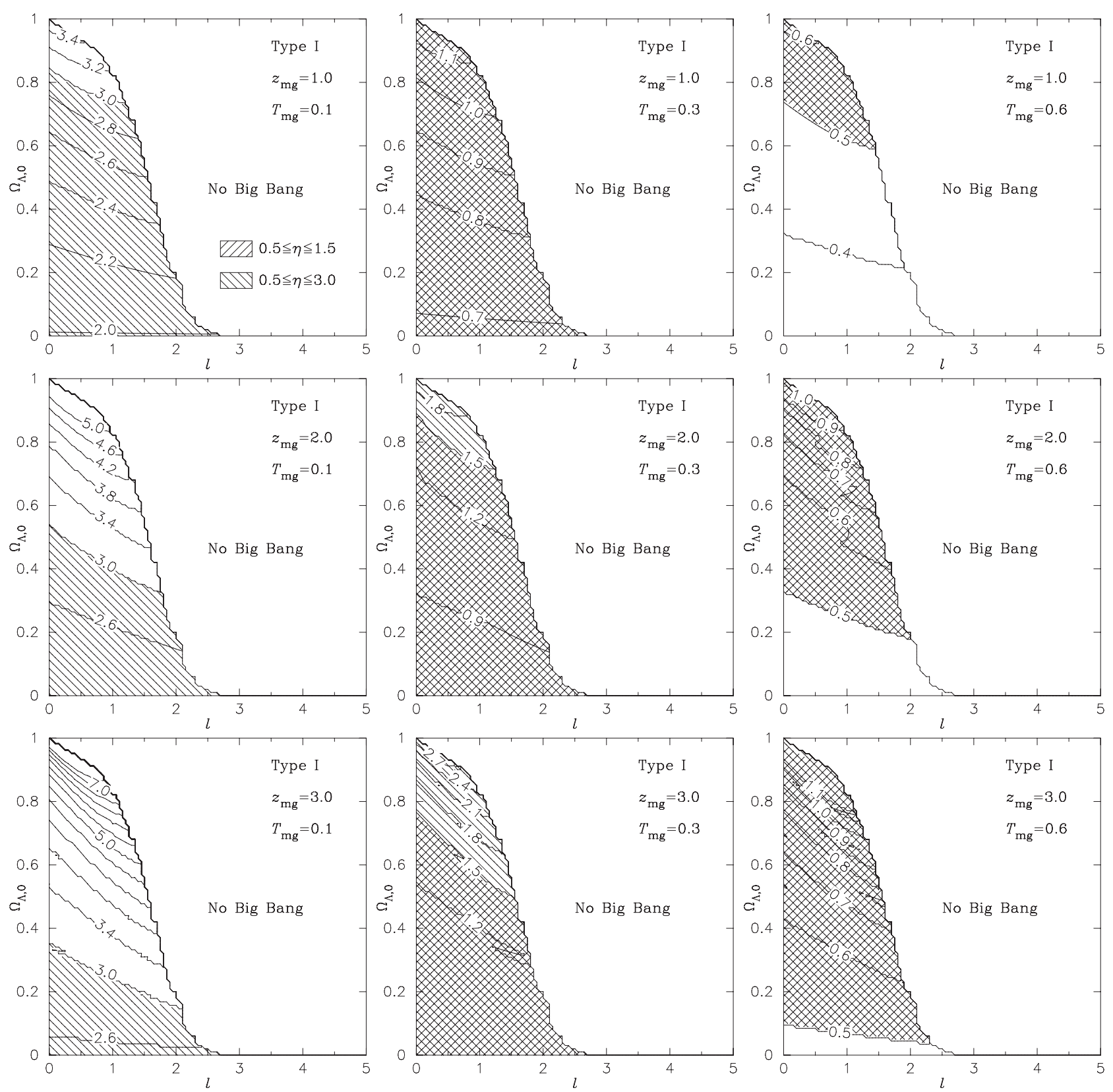

Fig. 2. The isopleths of the merging parameter $\eta$ for the type I models $\left(\Lambda \propto \tau^{-l}\right)$ : the ordinate of each diagram indicates $\Omega_{\Lambda, 0}$, and the abscissa is the decay parameter $l$. The three diagrams in the topmost row are for $z_{\mathrm{mg}}=1.0$, those in the middle row are for $z_{\mathrm{mg}}=2.0$, and those in the bottom row are for $z_{\mathrm{mg}}=3.0$. Three diagrams in each row correspond from left to right to $T_{\mathrm{mg}}=0.1,0.3$, and 0.6 , respectively. The domains for which no Big Bang occurs are indicated with a sign "No Big Bang". Furthermore, the domains for $0.5 \lesssim \eta \lesssim 1.5$ which we presume to be realistic based on Martini (2001); Totani \& Yoshii (2000); Totani et al. (2001); Cohen et al. (2003) are shown by hatching lines running diagonally to the upper right, and those for $0.5 \lessgtr \eta \lesssim 3.0$ by hatching lines running diagonally to the lower right.

Here, $V$ is the comoving volume and $\phi_{i}$ is the luminosity function of the galaxies of the $i$ th morphological Hubble type, for which results obtained through the $2 \mathrm{dF}$ Survey (Folkes et al. 1999) are adopted. The effect of galaxy merger is then incorporated into the above expression to get the final results.

\subsection{Galaxy merging}

The most popular contemporary scenario for large scale structure formation is hierarchical clustering due to CDM. According to this, formation is initiated in smaller scales, and then proceeds to larger scales to eventually produce galaxies and clusters of galaxies (Fukushige \& Makino 1997). From this point-of-view, it is critical to consider the effects of galaxy merger in the $N-m$ relation computations (Koo 1986). We therefore assume that the galaxy number evolution takes the form $\phi^{*}(z) \propto(1+z)^{\eta}$ in the Schechter parameterization (Rocca-Volmerange \& Guiderdoni 1990), and that $L^{*}(z) \propto(1+z)^{-\eta}$ in order to conserve the luminosity density, or, $\phi^{*}(z) \times L^{*}(z)=$ constant. Here, $\eta$ is a galaxy merger parameter (however, see the discussion below). This parameterization is based on the premise that the luminous flux 

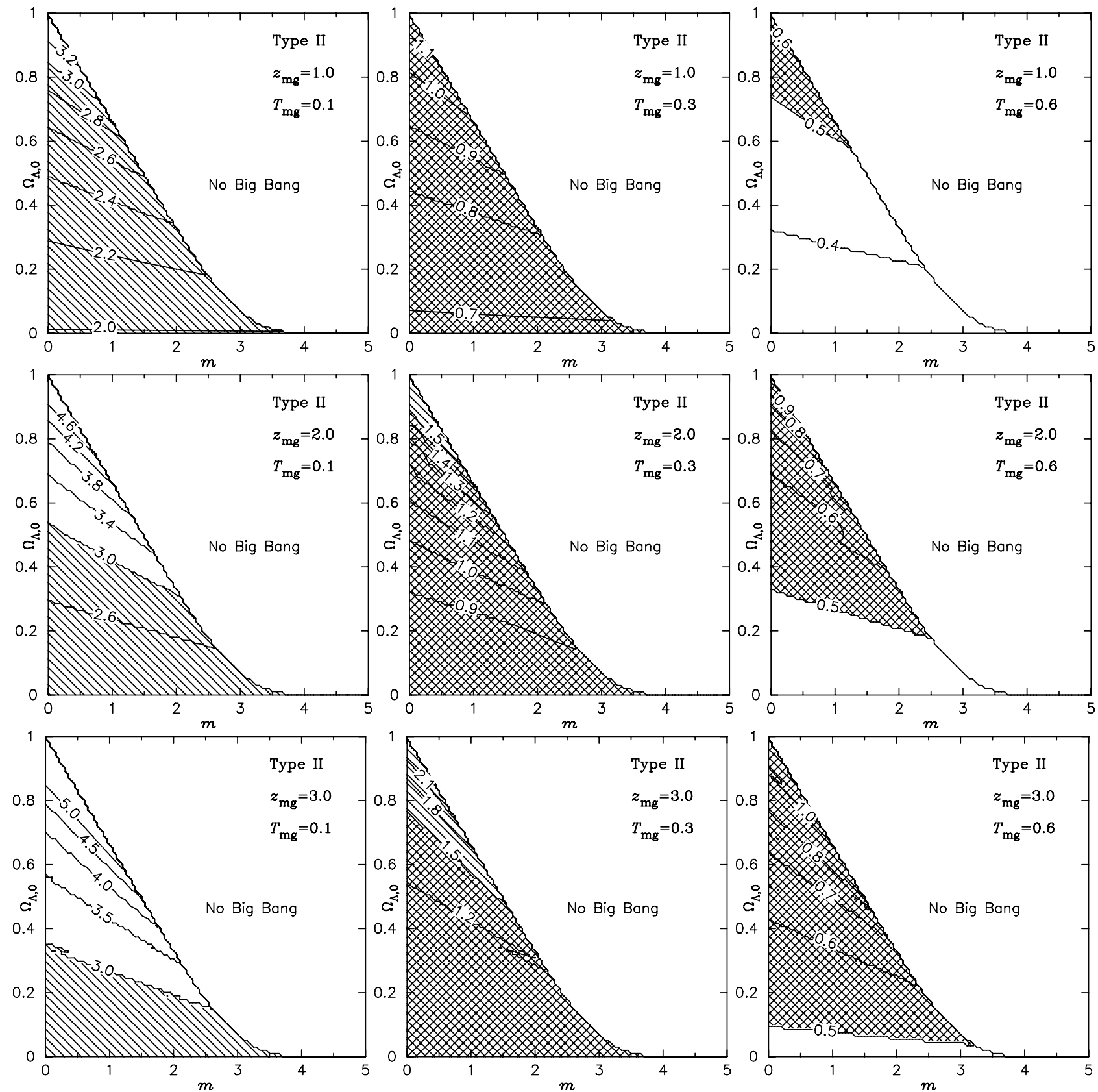

Fig. 3. The isopleths of the merging parameter $\eta$ for the type II models $\left(\Lambda \propto a^{-m}\right)$ : the same as Fig. 2, but for the type II models.

emitted by a contemporary galaxy should be approximated with the sum of the luminous fluxes given off by individual lesser-sized galaxies that have merged sometime in the past to form the contemporary one. The value of $\eta$ is usually believed to lie in the range $0.5 \lesssim \eta \lesssim 1.5$ on the basis of comparison with observations coupled with the use of the FriedmannLemître models (Martini 2001; Totani \& Yoshii 2000; Totani et al. 2001; Cohen et al. 2003). Nevertheless, no clear-cut consensus on a legitimate value of this parameter has yet been reached because of the number of astrophysical assumptions made for its determination. In the present work, we therefore adopt the range of $0 \leq \eta \leq 3.0$ thereby allowing for a wider margin for this quantity in view of the different types of cosmological models employed in the study.

It is therefore interesting to attempt to determine the value of $\eta$ solely on a cosmological basis. In other words, we would like to incorporate this quantity $\eta$ in the present work not as a free parameter but as a variable dependent on the other cosmological parameters.

Following the procedure of Patton et al. (2000, 2002), we can now relate the merging fraction $f_{\mathrm{mg}}$, which designates the number of galaxies undergoing mergers relative to the total number of galaxies which exist, to the cosmological variables.

A merging of a given pair of galaxies is assumed to have been completed in a timescale of $T_{\mathrm{mg}}$ Gyr. Assuming that every galaxy was in the form of 2-tuples, and if $n$-cycles of the merging process have been completed since the epoch of the redshift $z_{\mathrm{mg}}$ when the merging began, up to the present epoch $(z=$ $0)$, we get

$N(z)=\frac{N(z=0)}{\prod_{k=1}^{n}\left(1-0.5 f_{\mathrm{mg}}\left(z_{k}\right)\right)}$. 

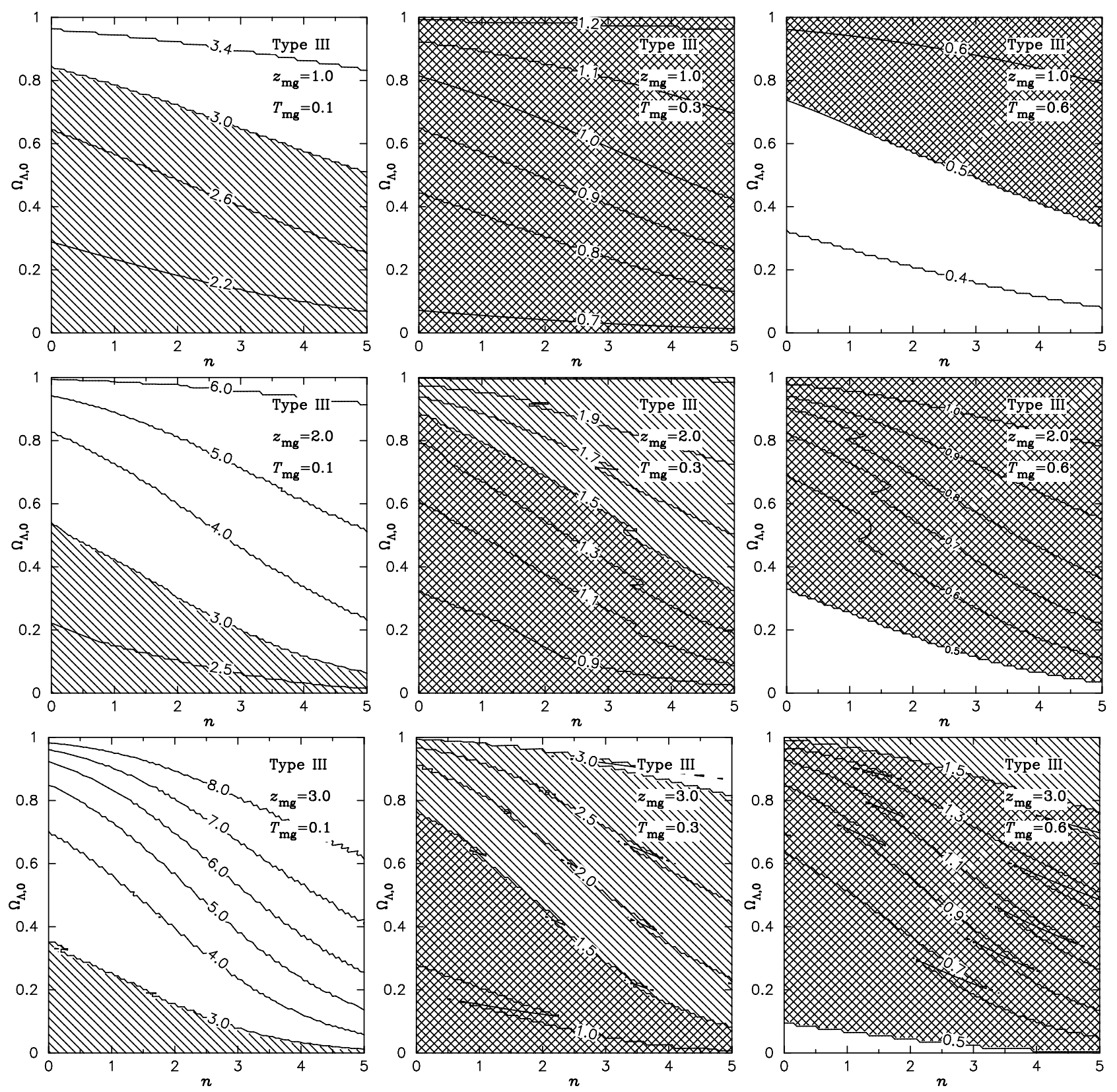

Fig. 4. The isopleths of the merging parameter $\eta$ for the type III models $\left(\Lambda \propto H^{n}\right)$ : The same as Fig. 2, but for the type III models. Note however that no domain is present for "No Big Bang". The reason for this is that the Big Bang inevitably takes place in this type of model for any combination of the parameter values unlike for the type I and II models.

We can use this to compute the redshift dependence of the number of galaxies. Here, we adopt the expression $f_{\mathrm{mg}}=$ $0.022(1+z)^{2.2}$ described by Cassata et al. (2005) to obtain Eq. (26). Provided that the number evolution of typical galaxies can be represented sufficiently well with $N(z)$ given above, we have $\phi^{*}(z) / \phi^{*}(z=0)=N^{*}(z) / N^{*}(z=0)$, so that we should be able to determine the value of $\eta$ by means of least squares fitting to the function $(1+z)^{\eta}$. The number of cycles of merging that should occur between $z=z_{\mathrm{mg}}$ and $z=0$ strongly depends on the value of $T_{\mathrm{mg}}$. This value, inferred from various numerical simulations as well as observational data, appears to indicate that $T_{\mathrm{mg}}$ is $\approx 0.5 \mathrm{Gyr}$ (Chatterjee 1993; Patton et al. 1997; Barnes 1988; Cohn et al. 2001; Gottlöber et al. 2002; Lu \& Wei 2003; Hernandez \& Lee 2004; Conselice et al. 2003). We have however varied the value of $T_{\mathrm{mg}}$ from 0.1 to 2.0 in our computations. Figures 2-4 show contour plots of $\eta$ obtained for the type I, II, and III models with several sets of employed values of the decay parameters and cosmological parameters under the assumptions mentioned above.

We begin with the behavior of $\eta$, one of the most crucial variables, as a function $T_{\mathrm{mg}}$ and $z_{\mathrm{mg}}$. As $T_{\mathrm{mg}}$ becomes smaller, the number of merging cycles increases so that the number of galaxies that might have existed in the past increases correspondingly, which in turn increases the value of $\eta$. This tendency is clearly noticeable in the diagrams in Figs. 2-4. Similarly, as $z_{\mathrm{mg}}$ gets larger, the merging can start farther back in the past. This means that we have a larger number of galaxy merging cycles which can take place up to the present epoch 
( $z=0$ ), leading to larger values of $\eta$ for the same reason mentioned above for a change in the $T_{\mathrm{mg}}$ value. However, the effect of a change in $z_{\mathrm{mg}}$ is not as large as that obtained by a change in $T_{\mathrm{mg}}$. In other words, the value of $\eta$ is extremely sensitive to the assumed value of $T_{\mathrm{mg}}$. It is interesting to note that in the case of $T_{\mathrm{mg}}=0.1 \mathrm{Gyr}$, we tend to get rather large values for $\eta$ falling outside the specified range $0 \lesssim \eta \lesssim 3.0$ so long as we impose the condition that $z_{\mathrm{mg}}>2.2$ and the cosmic age is higher than 13 Gyr.

Next, we examine the dependencies of $\eta$ on the cosmological parameter $\Omega_{\Lambda, 0}$ and the $\Lambda$-decay parameter $l, m$, or $n$. It can be noticed that $\Omega_{\Lambda, 0}$ is the sole free cosmological model parameter, since we are concerned here with flat cases only. Figures 2-4 indicate that as the value of $\Omega_{\Lambda, 0}$ increases, the value of $\eta$ increases as well for the reason that an increase in $\Omega_{\Lambda, 0}$ makes the cosmic age corresponding to a given redshift $z$ higher. This in turn yields a larger number of galaxy merger cycles thereby leading to an increase in $\eta$. We also note that the cosmic age tends to become higher as the value of a decay parameter $l, m$, or $n$ is increased. This gives rise to a larger value of $\eta$ for the same reason as applicable to an increase in the value of $\Omega_{\Lambda, 0}$.

\section{Comparisons with observational data}

\subsection{Constraints from cosmic age considerations}

The lower limit set for the cosmic age is, perhaps, the most fundamental and compulsory restriction that must be surpassed by any realistic model universe. The cosmic ages determined by observations of metal-deficient stars are in some cases $14 \mathrm{Gyr}$ or higher, although the measurement errors tend to be rather large. In fact, the age of a metal-poor halo star, CS 22892-052 for instance, has been estimated to be $17 \pm 4 \mathrm{Gyr}$ (Cowan et al. 1997), $15.6 \pm 4.6$ Gyr (Cowan et al. 1999), or $14.2 \pm 3$ Gyr (Sneden et al. 2003) based on abundance analyses. Similarly, an age of $15.6 \pm 4$ Gyr has been indicated for HD 115444 (Westin et al. 2000). Furthermore, the age of CS 31082-001 is now estimated to be around 15.9-16.3 Gyr using a Uranium dating technique (Rengel et al. 2002) and 15.5 $\pm 3.2 \mathrm{Gyr}$ based on a different dating technique which is believed to be more reliable than with the Uranium (Schatz et al. 2002). There is also some indication that the age of HD 186478 may well exceed 18 Gyr (Johnson \& Bolte 2001).

In this study, we adopt $13 \mathrm{Gyr}$ as the lower limit for the age of our universe for the following reasons. The lower bound indicated by the observed ages of old stars is approximately $11 \mathrm{Gyr}$ as shown above, to which we need to add at least $2 \mathrm{Gyr}$ of elapsed time since the Big Bang until the commencement of star formation. Thus, it would be gratifying if we could identify models whose ages are sufficiently higher than this lower bound.

Figure 6 shows the isochrones of the cosmic ages of the models of each type as functions of $\Omega_{\Lambda, 0}$ and the decay parameters $l, m$, or $n$ for three cases of the galaxy merger timescale $T_{\mathrm{mg}}, 0.1,0.3$, and $0.6 \mathrm{Gyr}$.

Also shown in the diagrams are gray-colored domains that are capable of yielding ages higher than or equal to $13 \mathrm{Gyr}$. We clearly notice from these figures that as the value of the decay parameter $l, m$, or $n$ becomes larger, the cosmic age becomes greater as well.

It must also be noted that the Big Bang ceases to occur if the values of the decay parameters $l$ and $m$ become sufficiently large for the type I and II models, so that the isochrones for different ages can intersect respectively with the Big Bang boundary.

\subsection{Galaxy number vs. magnitude relation}

A large number of observations of the $N-m$ relations of galaxies have thus far been carried out covering magnitude ranges of 20 30 (Jarvis \& Tyson 1981; Koo 1986; Infante et al. 1986; Tyson 1988; Metcalfe et al. 1991; Jones et al. 1991; Metcalfe et al. 2001; Kümmel \& Wagner 2001). We have made comparisons between theoretical predictions and observations for the $B_{\mathrm{J}}$-magnitude to determine the values of $\Omega_{\Lambda, 0}$, and the decay parameters $l, m$, and $n$ specifying our $\Lambda$-decaying models, together with the value of $\eta$, assuming the Hubble parameter $h=0.7$ and the curvature parameter $\Omega_{\mathrm{K}, 0}=0$. The results are summarized in Fig. 5. Three rows, each of which consists of three panels corresponding to $z_{\mathrm{mg}}=1.0,2.0$, and 3.0, are for type I, II, and III models from top to bottom, respectively. In each panel, three best-fit cases based on the $\chi^{2}$ tests (as discussed later) are shown for $T_{\mathrm{mg}}=0.1,0.3$, and $0.6 \mathrm{Gyr}$ designated by $\mathrm{I}_{\mathrm{a}, \mathrm{b}, \mathrm{c}}, \mathrm{I}_{\mathrm{d}, \mathrm{e}, \mathrm{f}}$, and $\mathrm{I}_{\mathrm{g}, \mathrm{h}, \mathrm{I}}$ respectively for the type I models, and similarly for the type II and type II models. Also shown in each panel are two $N-m$ relation curves obtained with the corresponding Friedmann-Lemaitre models for $T_{\mathrm{mg}}=0.3$ and 0.6 Gyr. The observational data are those of (Jarvis \& Tyson 1981; Koo 1986; Infante et al. 1986; Tyson 1988; Metcalfe et al. 1991; Jones et al. 1991; Metcalfe et al. 2001; Kümmel \& Wagner 2001).

It can be seen in each panel that the theoretical curves given by the $\Lambda$-decaying models of all types and those for the Friedmann-Lemaître models cross each other at about a magnitude of 24. It can also be noticed that the portion of each of the Friedmann-Lemaittre curves for magnitudes brighter than 24 tends to overestimate the number of galaxies, while the opposite is true by a factor of more than five for magnitudes fainter than 24 . In the cases of the $\Lambda$-decaying models, on the other hand, the degrees of both the overestimates and the underestimates of galaxy numbers are significantly reduced. It must be mentioned, however, that the cosmic ages of all of these $\Lambda$-decaying models are higher than $16.5 \mathrm{Gyr}$ as can be found from Fig. 6 . Yet, we know that the $\Lambda$-decaying models, whose ages are as high as this, cannot be compatible with the observed CMBR spectra (Komiya et al. 2005).

In order to make a systematic comparison between theoretical predictions and the observed data for the $N-m$ relationship, we apply an optimization procedure using the $\chi^{2}$ test, where $\chi^{2}$ is defined as

$\chi^{2} \equiv \sum_{i}\left(\frac{N_{i, \mathrm{cal}}-N_{i, \mathrm{obs}}}{\sigma_{i}}\right)^{2}$

Here, $N_{i, \text { cal }}, N_{i, \text { obs }}$, and $\sigma_{i}$ are respectively the galaxy number counts predicted theoretically, those obtained from 

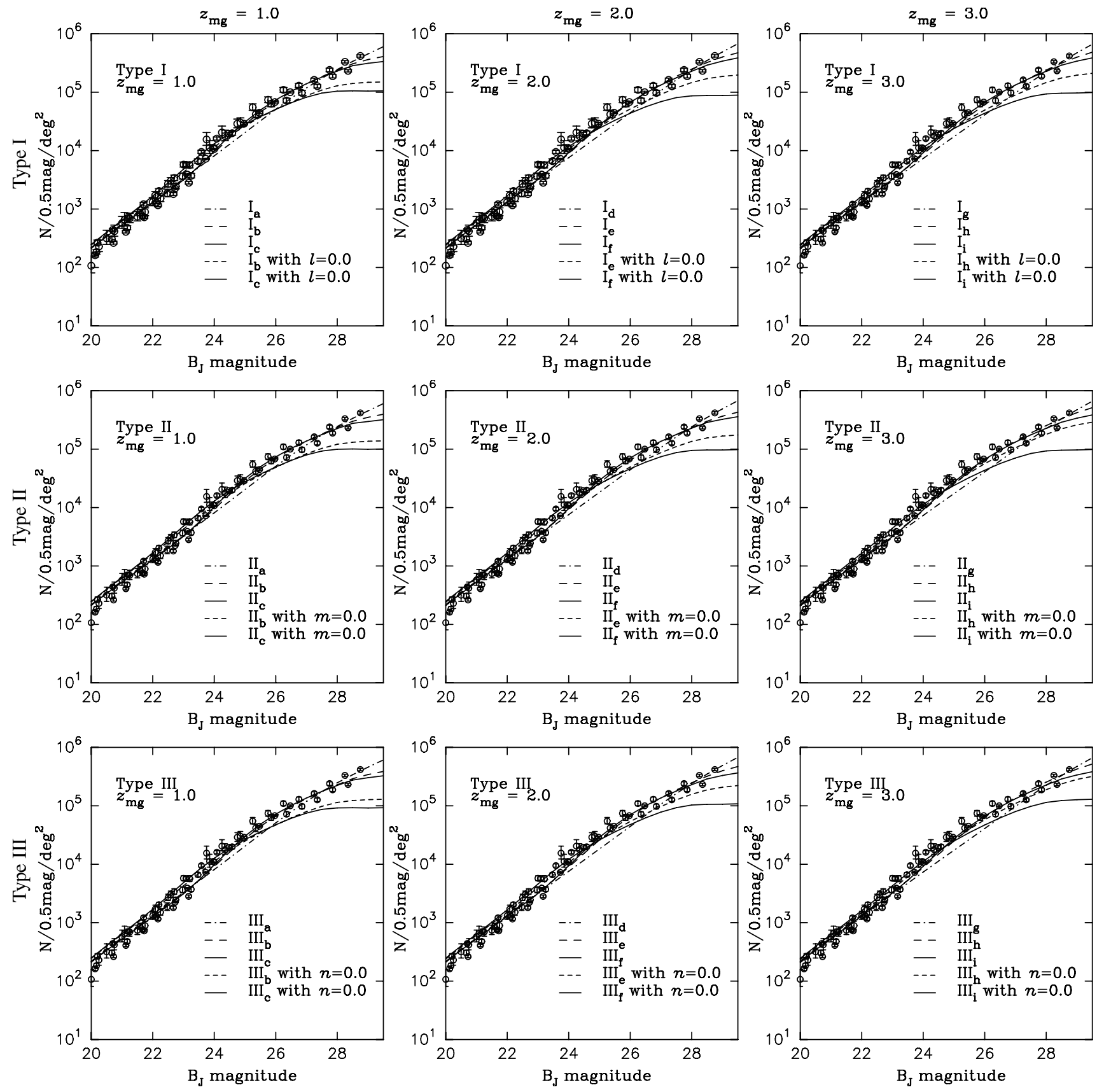

Fig. 5. Comparisons of the observed $N-m$ relation with those computed for the best-fit parameter values given in the Table 1: the ordinate of each diagram indicates the number counts per 0.5 mag interval per unit square degree of the sky, while the abscissa indicates apparent magnitudes of galaxies in the $B_{\mathrm{J}}$ band. The diagrams shown in the topmost row are for the type I models, those in the middle row are for the type II models, and those in the bottom row are for the type III models. The three diagrams in each row correspond from left to right to $z_{\mathrm{mg}}=1.0,2.0$ and 3.0, respectively. Also shown for the purpose of comparison are the results based on the flat Friedmann-Lemaitre models computed using the parameter values given in Table 1 except that those for the decay parameters are set equal to 0 .

observations, and the errors of the observed counts. We locate the minimum value $\chi_{\min }^{2}$ of the $\chi^{2}$ s, to find the domains in which the values of $\Delta \chi^{2} \equiv \chi^{2}\left(\Omega_{\Lambda, 0},\{l, m, n\}\right)-$ $\chi^{2}\left(\Omega_{\Lambda, 0, \min },\left\{l_{\min }, m_{\min }, n_{\min }\right\}\right)$ are less than or equal to the prescribed values, where $\Omega_{\Lambda, 0, \min }, l_{\min }, m_{\min }$, and $n_{\min }$ are the bestfit parameters that minimize the value of $\chi^{2}$.

Table 1 shows the best-fit values obtained by the $\chi^{2}$ test for the parameters $z_{\mathrm{mg}}, T_{\mathrm{mg}}, l, m, n, \Omega_{\Lambda, 0}$, and $\eta$, together with the resulting values of the cosmic age. It can be seen that the cosmic age is strongly dependent on the value of $T_{\mathrm{mg}}$ irrespective of the model type. This may be interpreted as follows. If the value of $T_{\mathrm{mg}}$ becomes larger, the value of $\eta$ becomes smaller. Under such circumstances, the model computations try to force the theoretical $N-m$ relation to agree as much as possible by further stretching the cosmic age. If, however, $T_{\mathrm{mg}}$ is less than, say, $0.1 \mathrm{Gyr}\left(\mathrm{I}_{\mathrm{a}}, \mathrm{I}_{\mathrm{d}}, \mathrm{I}_{\mathrm{g}}, \mathrm{II}_{\mathrm{a}}, \mathrm{II}_{\mathrm{d}}, \mathrm{II}_{\mathrm{g}}, \mathrm{III}_{\mathrm{a}}, \mathrm{III}_{\mathrm{d}}, \mathrm{III}_{\mathrm{g}}\right.$ in Table 1), 

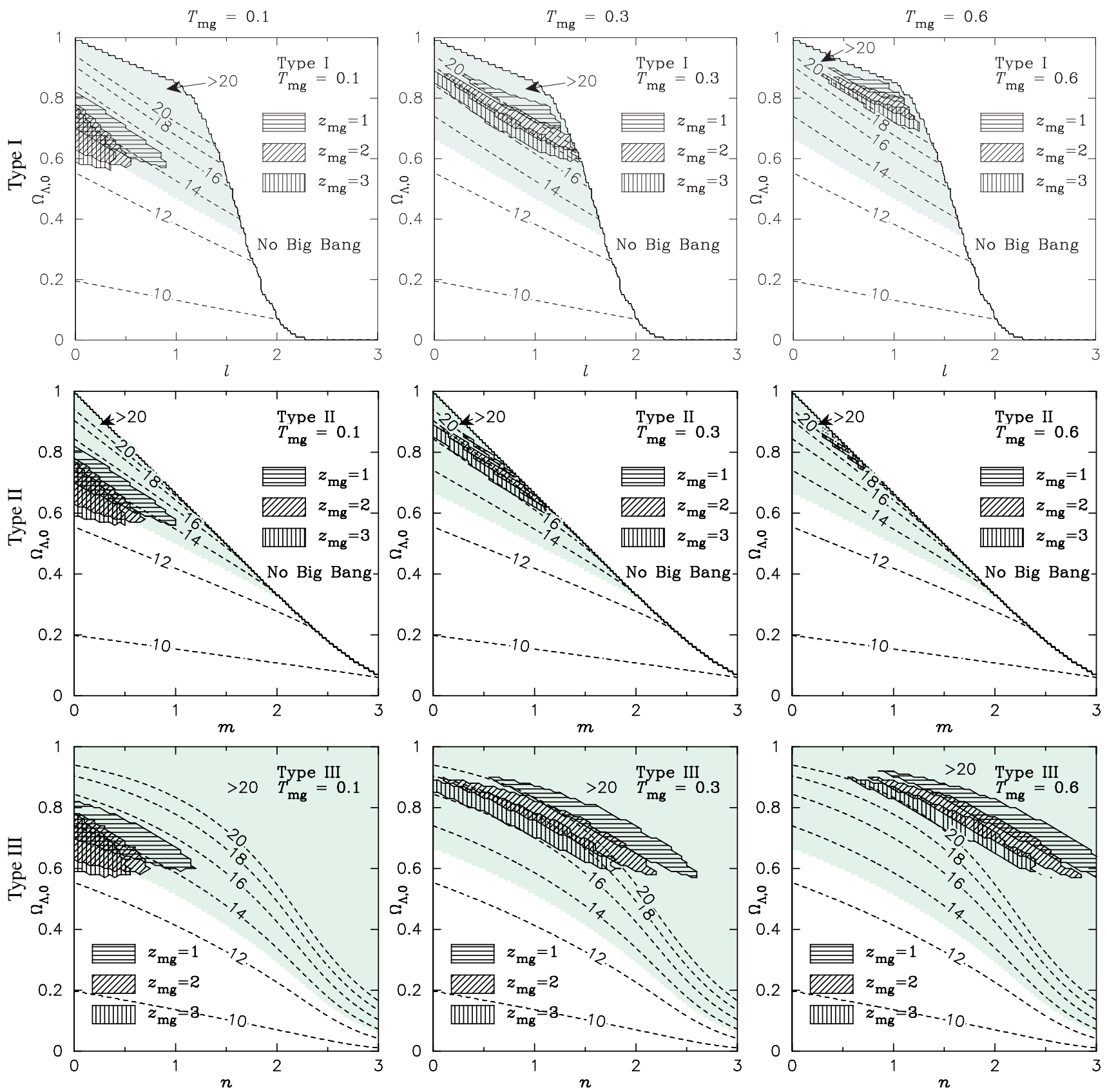

Fig. 6. The $3 \sigma$ confidence regions in the $\Omega_{\Lambda, 0}$ vs. decay parameters $l$, $m$, or $n$-space for each model type based on likelihood analyses: the ordinate of each diagram is $\Omega_{\Lambda, 0}$, while the abscissa indicates the decay parameter $l, m$, or $n$. The three diagrams in the topmost row are for the type I models, those in the middle row are for the type II models, and those in the bottom row are for the type III models. The diagrams on each row correspond from left to right to $T_{\mathrm{mg}}=0.1,0.3$ and 0.6 , respectively. In addition, the $3 \sigma$ regions for $z_{\mathrm{mg}}=1.0,2.0$ and 3.0 are hatched respectively with horizontal, diagonal, and vertical lines in each diagram. Also shown are the isochrones of the cosmic ages and the domains for which "no Big Bang" occurs. Furthermore, the domains for which the cosmic age surpasses 13 Gyr are indicated in gray color. As has already been mentioned, all of the type III models have Big Bang events.

then the number of merging cycles that occur becomes so large that the effect of the mergers becomes substantially dominant compared to the effects of the other parameters. Consequently, decreasing the value of the cosmological term further will have virtually no effect. Due to this reason, the FriedmannLemaitre model with a constant cosmological term can then be the best-fit model. Nevertheless, the resulting value of $\eta$ turns out to be appreciably different from the values determined by
Martini (2001); Totani \& Yoshii (2000); Totani et al. (2001); Cohen et al. (2003).

Figure 6 shows the $3 \sigma$ confidence regions obtained for each type of model on the basis of the $\chi^{2}$ values (Press et al. 1992). The isochrones of the cosmic age are shown also in the same diagrams, and the domains for ages higher than or equal to $13 \mathrm{Gyr}$ are indicated in gray color in the figure. As a common characteristic, we notice that the ranges permitted for the present-day 
Table 1. The most likely parameter values for each type of model for three values of $z_{\mathrm{mg}}$ and three values of $T_{\mathrm{mg}}$ : the columns in each table indicate, from left to right, the redshift $z_{\mathrm{mg}}$ at which the galaxy merger starts, the merging timescale for a pair of galaxies $T_{\mathrm{mg}} \mathrm{Gyr}$, the decay parameter $(l, m$, or $n)$, the present-day value of the cosmological term, the galaxy merging parameter $\eta$, and the resulting cosmic age in units of Gyr, respectively. The value of the curvature is assumed to be 0 .

\begin{tabular}{|c|c|c|c|c|c|c|}
\hline \multicolumn{7}{|c|}{ Type I } \\
\hline & $z_{\mathrm{mg}}$ & $T_{\mathrm{mg}}$ & $l$ & $\Omega_{\Lambda, 0}$ & $\eta$ & Age \\
\hline $\mathrm{I}_{\mathrm{a}}$ & 1.0 & 0.1 & 0.00 & 0.76 & 2.800 & 14.3 \\
\hline $\mathrm{I}_{\mathrm{b}}$ & 1.0 & 0.3 & 0.90 & 0.77 & 1.045 & 21.9 \\
\hline$I_{c}$ & 1.0 & 0.6 & 1.00 & 0.82 & 0.556 & 28.7 \\
\hline$\overline{I_{d}}$ & 2.0 & 0.1 & 0.00 & 0.72 & 3.492 & 13.7 \\
\hline $\mathrm{I}_{\mathrm{e}}$ & 2.0 & 0.3 & 0.75 & 0.75 & 1.464 & 18.8 \\
\hline $\mathrm{I}_{\mathrm{f}}$ & 2.0 & 0.6 & 1.15 & 0.77 & 0.804 & 25.8 \\
\hline $\mathrm{I}_{\mathrm{g}}$ & 3.0 & 0.1 & 0.00 & 0.67 & 3.833 & 13.1 \\
\hline $\mathrm{I}_{\mathrm{h}}$ & 3.0 & 0.3 & 0.75 & 0.71 & 1.731 & 17.2 \\
\hline $\mathrm{I}_{\mathrm{i}}$ & 3.0 & 0.6 & 1.20 & 0.73 & 1.026 & 23.1 \\
\hline \multicolumn{7}{|c|}{ Type II } \\
\hline & $z_{\mathrm{mg}}$ & $T_{\mathrm{mg}}$ & $m$ & $\Omega_{\Lambda, 0}$ & $\eta$ & $\mathrm{Age}$ \\
\hline$\overline{\mathrm{II}_{\mathrm{a}}}$ & 1.0 & 0.1 & 0.00 & 0.76 & 2.800 & 14.3 \\
\hline $\mathrm{II}_{\mathrm{b}}$ & 1.0 & 0.3 & 0.70 & 0.75 & 1.043 & 20.7 \\
\hline $\mathrm{II}_{\mathrm{c}}$ & 1.0 & 0.6 & 0.55 & 0.81 & 0.556 & 24.4 \\
\hline $\mathrm{II}_{\mathrm{d}}$ & 2.0 & 0.1 & 0.00 & 0.72 & 3.492 & 13.7 \\
\hline $\mathrm{II}_{\mathrm{e}}$ & 2.0 & 0.3 & 0.65 & 0.74 & 1.465 & 18.3 \\
\hline $\mathrm{II}_{\mathrm{f}}$ & 2.0 & 0.6 & 0.70 & 0.76 & 0.805 & 23.1 \\
\hline $\mathrm{II}_{\mathrm{g}}$ & 3.0 & 0.1 & 0.00 & 0.67 & 3.833 & 13.1 \\
\hline $\mathrm{II}_{\mathrm{h}}$ & 3.0 & 0.3 & 0.40 & 0.78 & 1.769 & 17.0 \\
\hline $\mathrm{II}_{\mathrm{i}}$ & 3.0 & 0.6 & 0.85 & 0.71 & 1.031 & 21.8 \\
\hline \multicolumn{7}{|c|}{ Type III } \\
\hline & $z_{\mathrm{mg}}$ & $T_{\mathrm{mg}}$ & $n$ & $\Omega_{\Lambda, 0}$ & $\eta$ & Age \\
\hline $\mathrm{III}_{\mathrm{a}}$ & 1.0 & 0.1 & 0.05 & 0.76 & 2.804 & $\overline{14.4}$ \\
\hline $\mathrm{III}_{\mathrm{b}}$ & 1.0 & 0.3 & 1.80 & 0.73 & 1.009 & 24.8 \\
\hline $\mathrm{III}_{\mathrm{c}}$ & 1.0 & 0.6 & 1.95 & 0.79 & 0.554 & 34.1 \\
\hline $\mathrm{III}_{\mathrm{d}}$ & 2.0 & 0.1 & 0.00 & 0.72 & 3.492 & 13.7 \\
\hline $\mathrm{III}_{\mathrm{e}}$ & 2.0 & 0.3 & 1.20 & 0.77 & 1.462 & 19.0 \\
\hline $\mathrm{III}_{\mathrm{f}}$ & 2.0 & 0.6 & 1.80 & 0.77 & 0.804 & 27.5 \\
\hline$\overline{\text { III }_{g}}$ & 3.0 & 0.1 & 0.00 & 0.67 & 3.833 & 13.1 \\
\hline $\mathrm{III}_{\mathrm{h}}$ & 3.0 & 0.3 & 0.80 & 0.79 & 1.771 & 17.3 \\
\hline $\mathrm{III}_{\mathrm{i}}$ & 3.0 & 0.6 & 1.75 & 0.73 & 1.027 & 23.8 \\
\hline
\end{tabular}

value of the cosmological term tend to become smaller as the value of the decay parameter $l, m$, or $n$ increases. The reason for this is that the cosmic age becomes higher as the value of the decay parameter is increased, leading to an increase in the spatial volume per unit redshift per unit square degree of the sky. Hence, the number of galaxies observed under the assumption of uniform spatial distribution of galaxies should also be higher, although we have an additional factor, viz., a time variation of the number density of galaxies due to galaxy mergers in computing the $N-m$ relation. This makes it easier for us to reduce, if not eliminate, the degree of discrepancy between the observed number of galaxies and the model predictions in the faint magnitude regions (the FBGP) cosmologically without recourse to other astrophysical agencies such as internal absorption (Wang 1991), the existence of dwarf galaxies (Abraham et al. 1996) etc.
Furthermore, we realize that the effect of the galaxy merger timescale $T_{\mathrm{mg}}$ is extremely large when compared to that of $z_{\mathrm{mg}}$. In the cases with $T_{\mathrm{mg}} \lesssim 0.1 \mathrm{Gyr}$, for example, we get values of $\eta$ much larger than 3.0 (see Figs. 2-4) due to the increase in the number of merging cycles, which in turn makes the galaxy number counts larger in the faint magnitude region in such a way as to reduce the value of $\Omega_{\Lambda, 0}$ required to account for the observations, yielding a cosmic age less than $13 \mathrm{Gyr}$.

It should also be pointed out that the variation of $z_{\mathrm{mg}}$ tends to shift the confidence region in a manner of a positive correlation in the $\Omega_{\Lambda, 0}$ vs. decay parameter $(l, m$, or $n)$-space. If we adopt a larger value for $z_{\mathrm{mg}}$ assuming that the galaxy mergers started in a more distant past, the galaxies that are present today should have undergone a larger number of mergers, thereby yielding a larger value of $\eta$. This implies that the effect of galaxy mergers can be more influential than the effects of the cosmological constant, decay parameter, and/or choices for the present day values of the cosmological parameters.

It should also be noted that the confidence regions for the type II models are considerably narrower than those of the other models. This reflects the fact that the type II models are highly sensitive to the value of the decay parameter $m$. On the other hand, the results are very degenerate against the variation of $z_{\mathrm{mg}}$, so that it is rather difficult, if not impossible, to distinguish between differences in the value of this parameter.

Finally, we would like to direct the reader's attention to the fact that the $3 \sigma$ regions found by the optimization procedure hardly contain the Friedmann-Lemaitre models $(l, m, n=0)$, which lie on the ordinates of Figs. 6. This leads us to the conclusion that the $\Lambda$-decaying models we are concerned with in the present work are far more advantageous from the stand-point of the $N-m$ relation than the Friedmann-Lemaître models.

\subsection{A consistency check using the results of the WMAP}

On account of the large number of model parameters involved, it is virtually impossible to determine their values unambiguously using just one type of observational data. Observations of CMBR anisotropy certainly provide us with another crucial test for the best-fit models derived in the preceding sections. It would therefore be essential to examine how well they compare with WMAP measurements, the highest precision data available for the CMBR anisotropy. For this purpose, we have carried out computations of the CMBR angular spectrum for a grid of type I models following the procedure described in Komiya et al. (2005). We have performed a grid-based parameter search assuming $h=0.7, \Omega_{\mathrm{K}, 0}=0$, while varying the value of the decay parameter $l$ from 0.0 to 5.0 with an interval of 0.02 , that of $\Omega_{\Lambda, 0}$ from 0.0 to 1.0 with an interval of 0.01 , that of $\Omega_{\mathrm{b}, 0}$ from 0.02 to 0.06 with an interval of 0.02 , that of the re-ionization optical depth $\tau$ from 0.05 to 0.20 with an interval of 0.05 , and that of the spectral index $n_{\mathrm{s}}$ from 0.95 to 1.05 with an interval of 0.05 . The likelihood code supplied by the WMAP team (Verde et al. 2003; Hinshaw et al. 2003) was used to obtain the likelihood functions of our theoretical spectra. We 


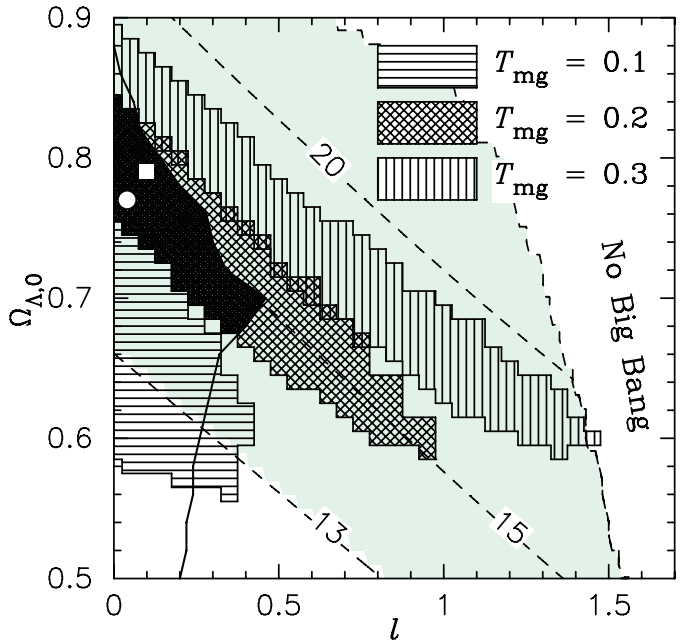

Fig. 7. $3 \sigma$-confidence regions on a $l-\Omega_{\Lambda, 0}$ plane obtained from analyses of $N-m$ relation and CMBR angular spectrum for type I models for $T_{\mathrm{mg}}=0.3$. The boundary of the confidence region found from the CMBR spectrum is indicated with a thick solid line. The $3 \sigma$-confidence regions from the $N-m$ relation analysis are for $T_{\mathrm{mg}}=$ $0.1,0.2$, and 0.3 in the case of $z_{\mathrm{mg}}=3.0$. The isochrones of the cosmic age are reproduced from Fig. 6, and the domain for which the cosmic age exceeds $13 \mathrm{Gyr}$ is colored gray. Also shown in the diagrams are the best-fit model for the $N-m$ relation (indicated with a square) and the corresponding one for the CMBR Spectrum (indicated with a circle), respectively.

have restricted the value of $\eta$ to be in the range between 0 and 3 as has already been mentioned. We note again that the confidence region of the $N-m$ relation is sensitively dependent on the value of $T_{\mathrm{mg}}$, and that this region, which contains the most-likely parameter values $\left(l=0.04, \Omega_{\Lambda, 0}=0.77\right)$ to simulate the observed CMBR spectrum, corresponds to $T_{\mathrm{mg}} \simeq 0.2$ if $z_{\mathrm{mg}}=3.0$ is assumed, as can be seen from Fig. 7. With $T_{\mathrm{mg}} \simeq 0.2$, the best-fit parameter set obtained for the $N-m$ relation is as follows: $l=0.1$ and $\Omega_{\Lambda, 0}=0.79$, from which we obtain $\eta=2.34$. We can dismiss the values of $T_{\mathrm{mg}} \leq 0.1$ for the reason that the resulting ages of our universe turn out to be less than 13 Gyr. For $T_{\mathrm{mg}}=0.3$, on the other hand, no overlapping region can be found between any confidence region from the $N-m$ relation analysis and that of the CMBR spectral analysis. Hence, we need to adopt $T_{\mathrm{mg}}=0.2$, the second-best value from the stand-point of the $N-m$ relation analysis and that of the CMBR spectral analysis. However, the resulting theoretical $N-m$ relations with $T_{\mathrm{mg}}=0.3$ as far as our eye-ball determination can be made. The model with $T_{\mathrm{mg}}=0.2$ that yields the best-fit to the observed $N-m$ relation (indicated in Fig. 7 with a square) has the following parameter values: $l=0.10$, $\Omega_{\Lambda, 0}=0.79$. On the other hand, the model that produces the best-fit to the WMAP measurements of the CMBR (indicated in Fig. 7 with a circle) is characterized with the following: $l=$ $0.04, \Omega_{\Lambda, 0}=0.77$.

We therefore adopt the following as the best-fit model to account for both the observed $N-m$ relation and the WMAP data of the CMBR, although it is conceivable that having somewhat different confidence regions might result if a more detailed analysis using a more sophisticated procedure such as

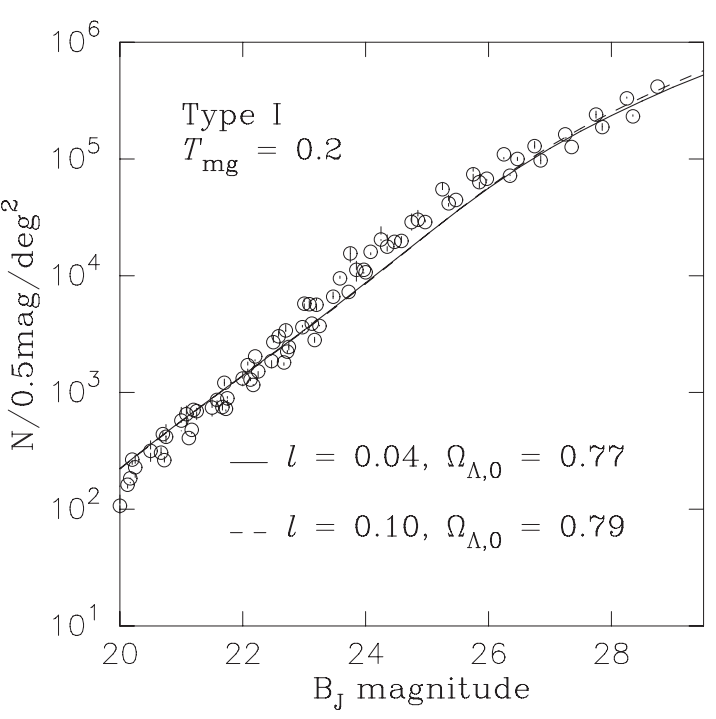

Fig. 8. Comparison of observational data for $N-m$ relation and theoretical curves computed for type I models. The ordinate is the galaxy number counts, and the abscissa is the $B_{\mathrm{J}}$ magnitude. The theoretical curves are the best-fit cases based on the $N-m$ relation only $(l=0.10$, $\left.\Omega_{\Lambda, 0}=0.79\right)$ and on the $\operatorname{WMAP}\left(l=0.04, \Omega_{\Lambda, 0}=0.77\right)$.

Markov chain Monte Carlo simulation is carried out without imposing any prior values for some of the parameters (we have assumed 0.7 for the value of the Hubble parameter $h$, and 0 for the curvature parameter $\left.\Omega_{\mathrm{K}, 0}\right): z_{\mathrm{mg}}=3.0, T_{\mathrm{mg}}=0.2, l=0.04$, $\Omega_{\Lambda, 0}=0.77, \eta=2.2269$. In this case, the cosmic age is 14.6 Gyr. In other words, the final model is much closer to a flat Friedmann-Lemaitre model than what we found solely from the $N-m$ relation analysis. It should be emphasized that the upper bound for the age of the type I model universe is $\sim 17.6 \mathrm{Gyr}$ in order to simulate the WMAP data of the CMBR spectrum as is readily seen in Fig. 7.

In Figs. 8 and 9, we show the $N-m$ relation and the CMBR spectra computed for some of the best-fit models. In particular, in the case of the $N-m$ relation analysis, since the parameter $\eta$ in this work is a dependent variable determined as a function of other parameters $\left(l, T_{\mathrm{mg}}, \Omega_{\Lambda, 0}\right)$, the role played by the $\Lambda$-decay in alleviating the FBGP is substantially reduced, which in turn gives rise to the $\eta$-dominated situation. As a consequence, our models gradually approach Friedmann-Lemaître models in the analysis of the $N-m$ relation. Nevertheless, we have demonstrated in this study that a time-decaying $\Lambda$ term should exist.

\section{Conclusions}

We have used cosmological models with a time-decaying cosmological term to compute the theoretical relationship between the apparent magnitude and the number count of galaxies (the $N-m$ relation) in order to make comparisons with observational data, and to provide constraints on the cosmological parameters as well as the galaxy merger parameters. The galaxy number density evolution is assumed to take the form $\phi^{*}(z) \propto(1+z)^{\eta}$, which yields $\phi^{*}(z) \times L^{*}(z)=$ constant under the condition that the luminosity density be conserved. 


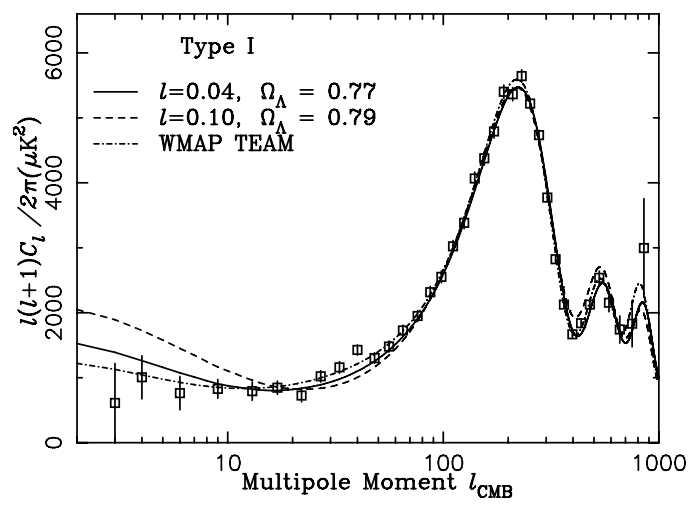

Fig. 9. Comparison of the WMAP observational data of the CMBR and theoretical spectra computed for type I models. The ordinate indicates temperature fluctuation while the abscissa indicates the multipoles. The theoretical curves are the best-fit cases corresponding to WMAP only $\left(l=0.04, \Omega_{\Lambda, 0}=0.77\right)$ or $N-m$ relation $(l=0.10$, $\Omega_{\Lambda, 0}=0.79$ ).

In contrast to the standard Friedmann-Lemitre model with a constant cosmological term, we have considered three types of variations for the cosmological term, $\Lambda \propto \tau^{-l}, a^{-m}, H^{n}$, and have performed systematic surveys on the decay parameters $l$, $m$, and $n$, the remaining cosmological parameters as well as the galaxy merging timescale $T_{\mathrm{mg}}$ and the redshift for the onset of galaxy formation $z_{\mathrm{mg}}$ in order to compute the likelihood functions based on the $\chi^{2}$ values for performing statistical analyses.

During the course of our analysis, we expressed the merging parameter $\eta$ in terms of the cosmological parameters and $T_{\mathrm{mg}}$. As a result, we found that the value of $\eta$ is strongly dependent on the assumed value of $T_{\mathrm{mg}}$; for $T_{\mathrm{mg}} \lessgtr 0.1$, for example, we get $\eta>3.0$, which is not compatible with the possible range of $0 \lesssim \eta \lesssim 3$.0. It is also of interest to note that for $T_{\mathrm{mg}} \lesssim 0.1$, the value of $\eta$ obtained from the $N-m$ relation computations becomes so large as to lead us to a cosmic age of less than $13 \mathrm{Gyr}$ irrespective of the presence of time variations for the cosmological term. For $T_{\mathrm{mg}} \gtrsim 0.6$, on the other hand, the models try to compensate for the reduced effect of $\eta$ by raising the cosmic age beyond 20 Gyr which is far higher than the upper limit inferred from our analysis of the observed angular spectrum of the CMBR (Komiya et al. 2005). Hence, we may safely bracket $T_{\mathrm{mg}}$ to be between $0.1 \mathrm{Gyr}$ and $0.6 \mathrm{Gyr}$.

In the case of $z_{\mathrm{mg}}=1.0$, either the value of $\eta$ becomes too large or the cosmic age exceeds $20 \mathrm{Gyr}$ as in the case with $T_{\mathrm{mg}} \gtrsim 0.6$ in order to simulate the observed $N-m$ relation. However, we must note that observations by Cassata et al. (2005) actually show that galaxy mergers are already taking place at $z=2.2$ or greater, so that $z_{\mathrm{mg}}=1.0$ is not quite compatible with the observational results. In general, the introduction of the time-decay of the cosmological term increases the cosmic age, which in turn enhances the number of galaxies fainter than the apparent magnitude 24 . This certainly helps to reduce the degree of the "faint blue galaxy problem (FBGP)" by bringing the theoretical curves of the $N-m$ relation into better agreement with observations especially for magnitudes larger than 24. A significant advantage of our $\Lambda$-decaying models may be the fact that their ages can be made higher than $14 \mathrm{Gyr}$, and they are less susceptible to the cosmic age problem. An important point to be stressed here is that a feed-back effect has now been incorporated into the model computations by relating the galaxy merging parameter $\eta$ to the value of a decay parameter $(l, m$, or $n)$ and those of the cosmological model parameters in such a way so that, for instance, the value of $\eta$ becomes too large if the cosmic age is too high, which can in turn suppress the cosmic age.

Based on our analysis of the $N-m$ relation, we have obtained the following as the best-fit models: $z_{\mathrm{mg}}=3.0, T_{\mathrm{mg}}=$ $0.3, l=0.75_{-0.75}^{+0.55} \Omega_{\Lambda, 0}=0.71_{-0.09}^{+0.17}, \eta=1.73_{-0.07}^{+0.14}$ and the cosmic age of $17.2_{-1.2}^{+1.3}$ for the type I model, $z_{\mathrm{mg}}=3.0, T_{\mathrm{mg}}=$ $0.3, m=0.40_{-0.40}^{+0.70}, \Omega_{\Lambda, 0}=0.78_{-0.17}^{+0.10}, \eta=1.77_{-0.12}^{+0.12}$, and the cosmic age of $17.0_{-1.1}^{+0.9}$ for the type II model, and $z_{\mathrm{mg}}=$ $3.0, T_{\mathrm{mg}}=0.3, n=0.80_{-0.80}^{+0.90}, \Omega_{\Lambda, 0}=0.79_{-0.17}^{+0.09}, \eta=$ $1.77_{-0.11}^{+0.13}$, and the cosmic age of $17.3_{-1.1}^{+1.1}$ for the type III model, respectively.

Mention must be made of the behavior of the scale factor for the type II $\left(\Lambda \propto a^{-m}\right)$ and III $\left(\Lambda \propto H^{n}\right)$ models which are found to be quite similar to each other from the present epoch down to the era of $z \sim 10$, as long as the values of their parameters are selected to simulate the observed $N-m$ relation. We shall have to deal with much higher redshift phenomena such as the Big Bang nuclear synthesis and the CMBR spectra to differentiate and assess the effects of the different types of the $\Lambda$-term decays.

In view of the cosmological importance of the WMAP observations, we have also made a comparison of the theoretical CMBR spectra computed for the type I models including the best-fit model inferred from our $N-m$ relation analysis given above. We find that somewhat different models than those found for the $N-m$ relation are required to account for the WMAP data. Consequently, we propose the following as a final best-fit model that is capable of simulating both types of observations sufficiently well: $z_{\mathrm{mg}}=3.0, T_{\mathrm{mg}}=0.2, l=0.04$, $\Omega_{\Lambda, 0}=0.77, \eta=2.2269$, which gives rise to a cosmic age of 14.6 Gyr. Obviously, this final model is significantly closer to a flat Friedmann-Lemaître model compared to the model deduced solely from the $N-m$ relation analysis. In spite of this outcome, the present work strongly indicates that there is a relatively slow decay of the cosmological term or the dark energy.

The matter creation rate in our type I model universe is $\sim 2.64 \times 10^{-16} l$ proton $/ \mathrm{cm}^{3} / \mathrm{yr}$ as can be readily shown with Eq. (5), which yields on the order of $10^{-17}$ proton $/ \mathrm{cm}^{3} / \mathrm{yr}$ for the final model. This value is far too low to be detectable with present-day observational techniques (Lima et al. 1996). We shall undertake a similar analysis shortly using type II and type III models to see how well they compare to the type I model.

Acknowledgements. We thank M. Morikawa and F. Takahara for providing useful information. Thanks are also due to J. Lim for useful discussions. The parameter sweep calculations were carried out on the P32 cluster of the AIST Super Cluster at the National Institute of Advanced Industrial Science and Technology.

\section{References}

Abraham, R. G., Tanvir, N. R., Santiago, B. X., et al. 1996, MNRAS, 279, L47 
Alcaniz, J. S., \& Maia, J. M. F. 2003, Phys. Rev. D, 67, 43502

Arimoto, N., \& Yoshii, Y. 1986, A\&A, 164, 260

Aurich, R., \& Steiner, F. 2003, Phys. Rev. D, 67, 123511

Barnes, J. E. 1988, ApJ, 331, 699

Barreiro, T., Bento, M. C., Santos, N. M. C., \& Sen, A. A. 2003, Phys. Rev. D, 68, 43515

Bloomfield Torres, L. F., \& Waga, I. 1996, MNRAS, 279, 712

Broadhurst, T. J., Ellis, R. S., \& Glazebrook, K. 1992, Nature, 355, 55

Caldwell, R. R., Dave, R., \& Steinhardt, P. J. 1998, Phys. Rev. Lett., 80, 1582

Cassata, P., Cimatti, A., Franceschini, A., et al. 2005, MNRAS, 357, 903

Chaboyer, B., Demarque, P., Kernan, P. J., \& Krauss, L. M. 1996, Science, 271, 957

Chatterjee, T. K. 1993, Ap\&SS, 199, 189

Chiba, T., Sugiyama, N., \& Nakamura, T. 1997, MNRAS, 289, L5

Coble, K., Dodelson, S., \& Frieman, J. A. 1997, PhRvD, 55, 1851

Cohen, S. H., Windhorst, R. A., Odewahn, S. C., Chiarenza, C. A., \& Driver, S. P. 2003, AJ, 125, 1762

Cohn, J. D., Bagla, J. S., \& White, M. 2001, MNRAS, 325, 1053

Conselice, C. J., Bershady, M. A., \& Dickinson, M. 2003, AJ, 126, 1183

Cowan, J. J., Mcwilliam, A., Sneden, C., \& Burris, D. L. 1997, ApJ, 480, 246

Cowan, J. J., Pfeiffer, B., Kratz, K. L., et al. 1999, ApJ, 521, 194

Cunha, J. V., ALcaniz, J. S., \& Lima, J. A. 2002, Phys. Rev. D, 66, 23520

Dev, A., Alcaniz, J. S., \& Jain, D. 2003, Phys. Rev. D, 67, 23515

Díaz-Rivera, L. M., \& Pimentel, L. O. 1999, Phys. Rev. D, 60, 123501

Driver, S. P., \& Phillipps, S. 1996, ApJ, 469, 529

Ferreira, P. G., \& Joyce, M. 1997, Phys. Rev. Lett., 79, 4740

Folkes, S., Ronen, S., Price, I., et al. 1999, MNRAS, 308, 459

Frieman, J. A., \& Waga, I. 1998, Phys. Rev. D, 57, 4642

Fukugita, M., Takahara, F., Yamashita, K., \& Yoshii, Y. 1990, ApJ, 361, L1

Fukushige, T., \& Makino, J. 1997, ApJ, 477, L9

Gardner, J. P. 1998, PASP, 110, 291

Gardner, J. P., Sharples, R. M., Carrasco, B. E., \& Frenk, C. S. 1996, MNRAS, 282, L1

Glazebrook, K., Peacock, J. A., Collins, C. A., \& Miller, L. 1994, MNRAS, 266, 65

Gottlöber, S., Kerscher, M., Kravtsov, A. V., et al. 2002, A\&A, 387, 778

Griffiths, R. E., Casertano, S., Ratnatunga, K. U., et al. 1994, ApJ, 435, L19

Guiderdoni, B., \& Rocca-Volmerange, B. 1990, A\&A, 227, 362

Hernandez, X., \& Lee, W. H. 2004, MNRAS, 347, 1304

Hinshaw, G., Spergel, D. N., Verde, L., et al. 2003, ApJS, 148, 135

Huterer, D., \& Turner, M. S. 2001, Phys. Rev. D, 64, 123527

Infante, L., Pritchet, C., \& Quintana, H. 1986, AJ, 91, 217

Jackson, J. C., \& Dodgson, M. 1998, MNRAS, 297, 923

Jarvis, J. F., \& Tyson, J. A. 1981, AJ, 86, 476

Johnson, J. A., \& Bolte, M. 2001, ApJ, 554, 888

Jones, L. R., Fong, R., Shanks, T., Ellis, R. S., \& Peterson, B. A. 1991, MNRAS, 249, 481

Kashino, Y., \& Kawabata, K. 1994, in Evolution of the Universe and its Observational Quest, ed. K. Sato (Tokyo: Universal Academy Press), 461

Kawabata, K., Matsushita, E., Komiya, Z., Hirano, K., \& Yamamoto, N. 2002, in Proc. IAU 8th Asian-Pacific Regional Meeting, Volume II, ed. S. Ikeuchi, J. Hearnshaw, \& T. Hanawa (Tokyo: The Astronomical Society of Japan), 263
Kogut, A., Spergel, D. N., Barnes, C., et al. 2003, ApJS, 148, 161

Komiya, Z., Kawabata, K., Hirano, K., Bunya, H., \& Yamamoto, N. 2005, J. Korean Astron. Soc., 38, 157

Koo, D. C. 1986, ApJ, 311, 651

Krauss, L. M., \& Chaboyer, B. 2003, Science, 299, 65

Kümmel, M. W., \& Wagner, S. J. 2001, A\&A, 370, 384

Lacey, C., \& Silk, J. 1993, ApJ, 402, 15

Lima, J. A. S., Germano, A. S. M., \& Abramo, L. R. W. 1996, Phys. Rev. D, 53, 4287

Lu, Y., \& Wei, J. 2003, Chin. J. Astron. Astrophys., 3, 395

Martini, P. 2001, AJ, 121, 598

Metcalfe, N., Shanks, T., Fong, R., \& Jones, L. R. 1991, MNRAS, 249, 498

Metcalfe, N., Shanks, T., Campos, A., McCracken, H. J., \& Fong, R. 2001, MNRAS, 323, 795

Mihara, K. 2001, PASJ, 53, 611

Morikawa, M. 1990, ApJ, 362, L37

Overduin, J. M., \& Cooperstock, F. I. 1998, Phys. Rev. D, 58, 43506

Patton, D. R., Pritchet, C. J., Yee, H. K. C., Ellingson, E., \& Carlberg, R. G. 1997, ApJ, 475, 29

Patton, D. R., Carlberg, R. G., Marzke, R. O., et al. 2000, ApJ, 536, 153

Patton, D. R., Pritchet, C. J., Carlberg, R. G., et al. 2002, ApJ, 565, 208

Peacock, J. A., Cole, S., Norberg, P., et al. 2001, Nature, 410, 169

Peebles, P. J. E., \& Ratra, B. 2003, Rev. Mod. Phys., 75, 559

Perlmutter, S., Aldering, G., Goldhaber, G., et al. 1999a, ApJ, 517, 565

Perlmutter, S., Turner, M. S., \& White, M. 1999b, Phys. Rev. Lett., 83, 670

Podariu, S., \& Ratra, B. 2000, ApJ, 532, 109

Press, W. H., Teukolsky, S. A., Vetterling, W. T., \& Flannery, B. P. 1992, Numerical Recipes in FORTRAN, 2nd edn. (New York: Cambridge University Press)

Rengel, M., Mateu, J., \& Bruzual, A. G. 2002, in Extragalactic Star Glusters, ed. D. Geisler, E. K. Grebel, \& D. Minniti (San Francisco: Astronomical Society of the Pacific), 716

Riess, A. G., Filippenko, A. V., Challis, P., et al. 1998, AJ, 116, 1009

Rocca-Volmerange, B., \& Guiderdoni, B. 1990, MNRAS, 247, 166

Sandage, A. 1961, ApJ, 133, 355

Sandage, A. 1965, ApJ, 141, 1560

Schatz, H., Toenjes, R., Pfeiffer, B., et al. 2002, ApJ, 579, 626

Shimasaku, K., \& Fukugita, M. 1998, ApJ, 501, 578

Silveira, V., \& Waga, I. 1994, Phys. Rev. D, 50, 4890

Silveira, V., \& Waga, I. 1997, Phys. Rev. D, 56, 4625

Sneden, C., Cowan, J. J., Lawlers, J. E., et al. 2003, ApJ, 591, 936

Spergel, D. N., Verde, L., Peiris, H. V., et al. 2003, ApJS, 148, 175

Teerikorpi, P. 2004, A\&A, 424, 73

Totani, T., \& Yoshii, Y. 2000, ApJ, 540, 81

Totani, T., Yoshii, Y., Maihara, T., Iwamuro, F., \& Motohara, K. 2001, ApJ, 559, 592

Turner, M. S., \& White, M. 1997, Phys. Rev. D, 56, R4439

Tyson, J. A. 1988, AJ, 96, 1

Verde, L., Peiris, H. V., Spergel, D. N., et al. 2003, ApJS, 148, 195

Viana, P. T. P., \& Liddle, A. R. 1998, Phys. Rev. D, 57, 674

Waga, I., \& Miceli, A. P. M. R. 1999, Phys. Rev. D, 59, 103507

Wang, B. 1991, ApJ, 383, L37

Westin, J., Sneden, C., Gustafsson, B., \& Cowan, J. J. 2000, ApJ, 530, 783

Yoshii, Y., \& Peterson, B. A. 1991, ApJ, 372, 8

Yoshii, Y., \& Sato, K. 1992, ApJ, 387, L7

Yoshii, Y., \& Takahara, F. 1988, ApJ, 326, 1 